\title{
Identification of predictive biomarkers of disease state in transition dairy cows
}

\author{
D. Hailemariam, ${ }^{\star}$ R. Mandal,† F. Saleem,† S. M. Dunn, ${ }^{\star}$ D. S. Wishart, $†$ and B. N. Ametaj ${ }^{\star 1}$ \\ *Department of Agricultural, Food and Nutritional Science, Edmonton, Alberta, Canada T6G 2P5 \\ †Departments of Computer and Biological Sciences, University of Alberta, Edmonton, Alberta, Canada T6G 2M9
}

\begin{abstract}
In dairy cows, periparturient disease states, such as metritis, mastitis, and laminitis, are leading to increasingly significant economic losses for the dairy industry. Treatments for these pathologies are often expensive, ineffective, or not cost-efficient, leading to production losses, high veterinary bills, or early culling of the cows. Early diagnosis or detection of these conditions before they manifest themselves could lower their incidence, level of morbidity, and the associated economic losses. In an effort to identify predictive biomarkers for postpartum or periparturient disease states in dairy cows, we undertook a cross-sectional and longitudinal metabolomics study to look at plasma metabolite levels of dairy cows during the transition period, before and after becoming ill with postpartum diseases. Specifically we employed a targeted quantitative metabolomics approach that uses direct flow injection mass spectrometry to track the metabolite changes in 120 different plasma metabolites. Blood plasma samples were collected from 12 dairy cows at 4 time points during the transition period ( -4 and -1 wh before and 1 and 4 wh after parturition). Out of the 12 cows studied, 6 developed multiple periparturient disorders in the postcalving period, whereas the other 6 remained healthy during the entire experimental period. Multivariate data analysis (principal component analysis and partial least squares discriminant analysis) revealed a clear separation between healthy controls and diseased cows at all 4 time points. This analysis allowed us to identify several metabolites most responsible for separating the 2 groups, especially before parturition and the start of any postpartum disease. Three metabolites, carnitine, propionyl carnitine, and lysophosphatidylcholine acyl C14:0, were significantly elevated in diseased cows as compared with healthy controls as early as 4 wk before parturition, whereas 2 metabolites, phosphatidylcholine acyl-alkyl C42:4 and phosphatidylcholine diacyl C42:6,
\end{abstract}

Received March 13, 2013.

Accepted January 16, 2014

${ }^{1}$ Corresponding author: burim.ametaj@ualberta.ca could be used to discriminate healthy controls from diseased cows 1 wk before parturition. A 3-metabolite plasma biomarker profile was developed that could predict which cows would develop periparturient diseases, up to 4 wk before clinical symptoms appearing, with a sensitivity of $87 \%$ and a specificity of $85 \%$. This is the first report showing that periparturient diseases can be predicted in dairy cattle before their development using a multimetabolite biomarker model. Further research is warranted to validate these potential predictive biomarkers.

Key words: predictive biomarker, plasma metabolite, periparturient disease, dairy cow

\section{INTRODUCTION}

In dairy cows, pathologies associated with parturition, such as mastitis, metritis, retained placenta, laminitis, ketosis, and milk fever, are very common (BigrasPoulin et al., 1990). The prevalence of these diseases is disproportionately high during the transition period, where 30 to $50 \%$ of the cows are affected by one or more of these conditions (LeBlanc, 2010). Periparturient diseases can significantly affect a dairy cow's production efficiency by reducing milk production, altering milk composition, reducing reproductive performance, increasing treatment costs, or reducing the life expectancy of the cow. Four conditions, metritis, infertility, mastitis, and laminitis, have been shown to account for the largest share of economic loss suffered by the dairy industry. For instance, early culling of dairy cows costs the Canadian dairy industry an estimated $\$ 200$ million a year, with $60 \%$ of the 160,000 cows culled in Canada in 2008 being afflicted with one or multiple of these 4 diseases (Ametaj et al., 2012).

The pathophysiology of postpartum diseases is complex, interrelated, and multifactorial, often driven by several interconnected risk factors. Despite major advances in the knowledge of their etiology, treatments for these kinds of diseases are often expensive, ineffective, or not cost-efficient. Therefore, early diagnosis or detection of those conditions before they manifest could reduce their incidence, level of morbidity, and the associated economic losses. 
Genomics, proteomics, and metabolomics technologies are increasingly being used to help identify disease and disease risk biomarkers. Given that little evidence exists for a genetic cause to these postpartum or periparturient diseases, it stands to reason that metabolite alterations may be more suitable to developing a predictive biomarker test. More than a decade ago, studies in dairy cows established that elevated ketone bodies, such as BHBA, acetone, and acetoacetate in milk, could serve as metabolic biomarkers for subclinical ketosis (Enjalbert et al., 2001). Prepartum NEFA and postpartum BHBA were both significantly associated with development of clinical disease, whereas postpartum serum NEFA concentration was most associated with the risk of developing displaced abomasum, clinical ketosis, metritis, or retained placenta during the first 30 DIM (Ospina et al., 2010). In another study, cows developing postpartum diseases had higher mean serum NEFA and lower plasma IGF-I concentrations prepartum when compared with healthy cows (Piechotta et al., 2012). Indeed, metabolomics has been shown to be a particularly effective platform for investigating the pathophysiology of pregnancy-associated diseases in humans, such as pre-eclampsia and pregnancy-induced diabetes, and identifying predictive biomarkers for those conditions (Bahado-Singh et al., 2012, 2013). In a previous study using a metabolomics approach, we reported unhealthy alterations of rumen metabolites with increasing proportions of cereal grains in the diet of dairy cows (Ametaj et al., 2010). Extending on those observations, our group used multiple metabolomics platforms to identify and quantify rumen metabolites, confirming detailed rumen metabolite alterations with increased grain proportions in the diet $(0,15,30$, and $45 \%$ barley grain on a DM basis), as well as temporal changes in the metabolite concentrations during each dietary period (Saleem et al., 2012). Moreover, feeding dairy cows diets rich in rumen-degradable carbohydrates and low in fiber led to lower rumen $\mathrm{pH}$ and large accumulation of rumen endotoxin; the latter was correlated with perturbations of plasma metabolites allied to carbohydrate and lipid metabolism (Zebeli et al., 2011). Nuclear magnetic resonance-based metabolomics elucidated the relationship between the metabolite profile and technological properties of bovine milk from 2 dairy breeds (Sundekilde et al., 2011) and revealed strong association between milk metabolites and SCC in bovine (Sundekilde et al., 2013). Moreover, Klein et al. (2012) reported that high concentrations of milk glycerophosphocholine along with a high ratio of glycerophosphocholine to phosphocholine could be used to aid in the reliable selection of healthy and metabolically stable cows for breeding purposes. Building from the success of these efforts, we decided to perform a longitudinal and cross-sectional metabolomic study to look at plasma metabolite levels of dairy cows during the transition period, before and after becoming ill with different postpartum pathologies. This was done to investigate whether changes in plasma metabolite concentrations could be predictive for the development of periparturient or postpartum diseases in dairy cows.

Specifically, we employed a targeted quantitative metabolomics approach that uses direct flow injection MS/MS to track the metabolite changes in 120 different plasma metabolites. Blood plasma samples were collected from 12 dairy cows at 4 time points during the transition period ( -4 and -1 wk before and 1 and 4 wk after parturition). Out of the 12 cows studied, 6 developed multiple periparturient diseases during the postcalving period, whereas the other 6 remained healthy during the entire experimental period. From this work, we were able to identify at least 5 plasma metabolites that can be used as potential predictive biomarkers for the early diagnosis and monitoring of multiple periparturient diseases in transition dairy cows.

\section{MATERIALS AND METHODS}

\section{Pre- and Postpartum Diets}

All cows were fed the same close-up diet starting at 3 wk before the expected day of parturition. The close-up diet is usually offered to the dairy cows when they are close to parturition and contained approximately $20 \%$ concentrate on a DM basis (Table 1). After parturition, cows were gradually switched during the first $7 \mathrm{~d}$ to a fresh lactation diet with a higher proportion of grain (up to $50 \%$ on a DM basis) to meet the energy demands for high milk production (Table 2). Daily ration was offered as TMR for ad libitum intake to allow approximately $10 \%$ feed refusals throughout the experiment. All cows were fed once daily in the morning at $0800 \mathrm{~h}$.

\section{Sample Collection}

Blood samples were collected from the coccygeal veins of 12 transition Holstein dairy cows at 4 time points: during 4 (25-31 d) and $1 \mathrm{wk}(4-10 \mathrm{~d})$ before calving (d 0) and during 1 (4-10 d) and 4 wk (25-31 d) after calving. Multiparous cows, with parities ranging from 2 to 4 , were used in the study. Samples were collected before the morning feeding at $0800 \mathrm{~h}$ using 10 $\mathrm{mL}$ Vacutainer tubes (Becton Dickinson, Franklin Lake, NJ) containing a sodium heparin anticoagulant. Blood samples were stored on ice and centrifuged within 20 min at $4^{\circ} \mathrm{C}$ for $20 \mathrm{~min}$ at $3,000 \times g$ in a Rotanta $460 \mathrm{R}$ centrifuge (Hettich Zentrifugan, Tuttlingen, Germany) to separate the plasma. Plasma samples were stored at 
Table 1. Prepartum diet for dry cows

\begin{tabular}{lc}
\hline Item & $\begin{array}{c}\text { Close-up } \\
\text { diet (CUD) }\end{array}$ \\
\hline Ingredient, \% of DM & \\
Alfalfa hay & 10.0 \\
Barley silage & 63.0 \\
Alfalfa silage & 00.0 \\
CUD grain & 27.0 \\
Nutrient composition of CUD grain, \% in $100 \mathrm{~kg}$ of mix & 55.0 \\
Barley grain, rolled & 7.5 \\
Canola meal & 6.2 \\
Dairy dry cow micro-premix & 8.7 \\
Limestone & 15.7 \\
Animate & 0.9 \\
Molasses & 4.1 \\
Canola oil & 1.7 \\
Yeast & \\
\hline
\end{tabular}

$-20^{\circ} \mathrm{C}$ until further analysis. All sick cows were diagnosed by a herd veterinarian.

Six cows were free of any diseases during the entire experimental period, whereas 6 different cows were diagnosed with one or more periparturient pathologies, including metritis, mastitis, laminitis, or retained placenta. All experimental procedures were approved by the University of Alberta Animal Policy and Welfare Committee for Livestock and animals were cared for in accordance with the guidelines of the Canadian Council on Animal Care (1993).

\section{Plasma Analyses}

A total of 48 plasma samples were analyzed using a targeted quantitative metabolomics approach employing a commercially available assay kit-based direct flow MS/MS technique (AbsoluteIDQ 180 Kit; Biocrates Life Sciences AG, Innsbruck, Austria). This kit assay is specifically adapted to work on a 4000 QTrap (Applied Biosystems/MDS Sciex, Foster City, CA) mass spectrometer and can be used for targeted identification and quantification of up to 160 different metabolites, including AA, acylcarnitines, glycerophospholipids, sphingolipids, and sugars. The method combines the derivatization and extraction of analytes with the selective mass spectrometric detection and quantification using multiple reaction monitoring pairs. Isotope-labeled internal standards are integrated in the kit plate filter for metabolite quantification.

The Absolute $I D Q 180$ kit contains a 96 deep-well plate with a filter attachment, along with reagents and solvents used to prepare the plate assay. The first 8 wells in the kit are used for reference calibration, with 1 blank, 3 zero samples (urea solution), 1 standard, and 3 quality control samples provided with each kit. All protocols were followed according the manufacturer's user manual. Prior to analysis, samples were left to thaw on ice $(\sim 30 \mathrm{~min})$. After thawing they were vortexed and centrifuged at $13,000 \times g$ at $4^{\circ} \mathrm{C}$ for $3 \mathrm{~min}$. Ten microliters of supernatant for each plasma sample was loaded on a filter paper and dried under a stream of nitrogen. Extraction of the metabolites was then achieved using methanol containing $5 \mathrm{mM}$ ammonium acetate. The extracts were analyzed using a 4000 QTrap (Applied Biosystems/MDS Sciex) mass spectrometer. A standard flow injection protocol consisting of two $20-\mu \mathrm{L}$ injections (one for the positive and one for the negative ion detection mode) was applied for all measurements. Multiple reaction monitoring detection was used for quantification. Software, which was included in the kit, was used to control the entire assay workflow, from sample registration to automated calculation of metabolite concentrations to the export of the data.

\section{Statistical Analysis}

All metabolomic data analyses were performed using MetaboAnalyst (Xia et al., 2009) and ROCCET (Xia et al., 2013). Recommended statistical procedures for metabolomic analysis were followed according to previously published protocols (Wishart, 2010, Xia et al., 2009). Compounds that were frequently $(>50 \%)$ below the limit of detection were removed from consideration; otherwise, a value of one-half the lower limit of detection was used to avoid zero-value entries. Log scaling was used for normalization of all metabolomic concentration data for each of the 4 different sampling intervals. Visual inspection of the data confirmed its normal or Gaussian distribution for each time period. For the purposes of the present study, all periparturient pathologies (mastitis, metritis, laminitis, and retained placenta) were grouped into one category (diseased). Of note, cows had more than one pathology during sampling and, because of the low number of diseased cows, no differentiation between various pathological 
Table 2. Ingredients of high grain ration fed to cows during early lactation

\begin{tabular}{|c|c|}
\hline Item & $\begin{array}{c}\text { Early } \\
\text { lactation diet }\end{array}$ \\
\hline \multicolumn{2}{|l|}{ Ingredient, $\%$ of DM } \\
\hline Alfalfa hay & 10.0 \\
\hline Barley silage & 40.8 \\
\hline Dairy supplement & 49.2 \\
\hline \multicolumn{2}{|c|}{$\begin{array}{l}\text { Nutrient composition of dairy supplement, } \\
\% \text { in } 100 \mathrm{~kg} \text { of mix (uless otherwise noted) }\end{array}$} \\
\hline ADE Pak Natural $E^{1}$ & 0.06 \\
\hline Ruminant TM Pak ${ }^{2}$ & 0.10 \\
\hline Selenium, 1,000 mg/kg & 0.07 \\
\hline Custom TM complex premix ${ }^{3}$ & 0.06 \\
\hline Dicalcium phosphate $21 \%$ & 1.25 \\
\hline Corn distillers grain & 10.0 \\
\hline Corn, ground & 25.0 \\
\hline Corn, rolled & 30.11 \\
\hline Vitamin D, $10,000 \mathrm{KIU} / \mathrm{kg}$ & 0.02 \\
\hline Diamond V XPC ${ }^{4}$ & 0.14 \\
\hline Megalac $^{5}$ & 1.00 \\
\hline Fermenten ${ }^{6}$ & 2.00 \\
\hline Limestone & 1.50 \\
\hline Mag Ox $56 \%^{7}$ & 0.37 \\
\hline Canola meal & 15.5 \\
\hline Hi bypass soy (Amino plus) ${ }^{8}$ & 2.75 \\
\hline Soybean meal, $47.5 \%$ & 6.50 \\
\hline Sodium bicarbonate & 1.00 \\
\hline Salt & 0.11 \\
\hline Pork tallow & 2.45 \\
\hline Biotin $2 \%$, Rovimix $\mathrm{H}-2^{9}$ & 0.01 \\
\hline ADM Vit E 405 Vegetable source $^{10}$ & 0.02 \\
\hline
\end{tabular}

\footnotetext{
${ }^{1}$ ADE-Pak Natural E: a premix containing vitamins $\mathrm{A}, \mathrm{D}_{3}$, and E.

${ }^{2}$ Ruminant TM Pak: a premix containing cobalt, copper, iodine, manganese, and zinc.

${ }^{3}$ Custom TM complex: a custom product supplying organic sources of cobalt, copper, manganese, and zinc.

${ }^{4}$ Diamond V XPC: concentrated yeast (Diamond V Mills, Cedar Rapids, IA).

${ }^{5}$ Megalac: calcium soaps (Church \& Dwight, Princeton, NJ).

${ }^{6}$ Fermenten: fermentation by-product (Arm and Hammer Animal Nutrition/Church \& Dwight).

${ }^{7} \mathrm{Mag}$ Ox 56\%: 56\% magnesium guarantee.

${ }^{8}$ Amino Plus: a high by-pass soy meal.

${ }^{9}$ DSM Nutritional Products (Parsippany, NJ).

${ }^{10} \mathrm{ADM}$ Vit E 405 Vegetable source: natural source of vitamin E (Archer Daniels Midland).
}

conditions was tested. Furthermore, we hypothesized that some metabolic commonalities for at least some of these conditions may exist. To perform a standard cross-sectional 2-group study, we classified all cows that eventually developed periparturient pathologies into one category (diseased cows) and all cows that remained healthy into another category (healthy cows) and compared these groups at each time point $(-4,-1$, 1 , and $4 \mathrm{wk}$ ). To perform the longitudinal or disease trajectory analysis, we performed a similar grouping but compared time-dependent metabolite changes within each group over the 8-wk period. Univariate analysis ( $t$-test) was performed to assess the differences of detected metabolites between the healthy controls and diseased cows. Principal component analysis (PCA), partial least squares discriminant analysis (PLS-DA), and variable importance of projection (VIP) were performed to identify those metabolites showing signifi- cant differences between healthy and diseased animals (in the cross-sectional study) and to identify different temporal metabolic trends followed by healthy and diseased animals (in the longitudinal study; Wishart, 2010). Principal component analysis is an unsupervised classification technique for transforming a complex collection of data points so that the important properties of the sample can be more simply displayed along the X- and Y-axes. Two clearly identified clusters on a PCA plot are indicative that significant metabolite differences exist between the 2 groups.

Partial least squares discriminant analysis was used to enhance the separation between the groups by summarizing the data into a few latent variables that maximize covariance between the response and the predictors. To minimize the possibility that the observed separation for a PLS-DA plot was due to chance, we performed permutation testing. This involved repeated 
(2,000 times) PLS-DA calculations using different random labeling of the samples. The fraction of randomly labeled PLS-DA calculations that exceed the separation score seen with the correctly labeled data provides a measure of the statistical significance or $P$-value. A significant $P$-value $(<0.05)$ indicates that the separation observed between the 2 groups is very unlikely to be due to chance. A VIP plot was used to rank the metabolites based on their importance in discriminating sick from control groups of cows. Metabolites with the highest VIP values are the most powerful group discriminators. Typically, VIP values greater than 2 are significant and VIP values greater than 4 are highly significant.

Biomarker profiles and the quality of the biomarker sets were determined using receiver-operator characteristic curves as calculated by the ROCCET web server (Xia et al., 2013). A receiver-operator characteristic curve shows how the sensitivity and specificity of a test or biomarker profile change as the classification decision boundary is varied across the range of available biomarker scores. Because receiver-operator characteristic curves depict the performance of a biomarker test over the complete range of possible decision boundaries, it allows the optimal specificity and associated sensitivity to be determined post hoc. Receiver-operator characteristic curves are often summarized into a single metric known as the area under the curve (AUC). The AUC can be interpreted as the probability that a diagnostic test or a classifier will rank a randomly chosen positive instance higher than a randomly chosen negative one. If all positive samples are ranked before negative ones (i.e., a perfect classifier), the AUC is 1.0. An AUC of 0.5 is equivalent to randomly classifying subjects as either positive or negative (i.e., the classifier is of no practical utility). A rough guide for assessing the utility of a biomarker based on its AUC is 0.9 to 1.0 $=$ excellent; 0.8 to $0.9=$ good; 0.7 to $0.8=$ fair; 0.6 to $0.7=$ poor; 0.5 to $0.6=$ fail .

\section{RESULTS AND DISCUSSION}

\section{Periparturient Diseases and Metabolomics Approach}

Of the 12 animals studied, 6 developed postpartum diseases. Among the sick cows, a total of 4 different pathologies were detected, including mastitis, metritis, retained placenta, and laminitis. Of the 6 cows with disease state, 2 cows had retained placenta, metritis, and laminitis, 1 had retained placenta and laminitis, 1 had metritis and laminitis, and 2 had mastitis only. Four of the 6 cows were affected by multiple conditions during the postpartum period. Diagnoses and treatments were performed by an experienced veterinarian using standard symptomatology and clinical criteria. Bovine mastitis is an IMI that is caused by a wide range of microorganisms, including gram-negative and gram-positive bacteria (Zadoks et al., 2011). Metritis is a uterine pathology that involves inflammation of all the uterine layers and is associated with reduced reproductive efficiency (Sheldon et al., 2006), whereas retained placenta is described as a failure to pass all or parts of placenta from the uterus within $24 \mathrm{~h}$ of calving. Laminitis is a metabolic disorder of the corium that affects the lamellae of the claw with sole hemorrhage and white line defects caused by improper nutrition or related to bacterial diseases, such as metritis or mastitis, and are associated with lameness (Kloosterman, 2007; Buch et al., 2011). Previous studies support the notion that periparturient diseases in dairy cows are interrelated and tend to occur as a complex especially during postpartum period (Curtis et al., 1983; Correa et al., 1993; Buch et al., 2011).

Metabolomic data were obtained for all 12 transition dairy cows at 4 different time points. An average of 120 different plasma metabolites were identified and quantified for each sample from each of the 6 healthy and 6 diseased cows (Table 3). These metabolites can be grouped into 5 categories (with the average number of compounds identified in brackets): amino acids (14), acylcarnitines (10), glycerophospholipids (80), sphingolipids (15), and hexose (1). Principal component analysis was used to reduce this large set of variables (120 metabolites) into 2 principal components (PCA 1 and 2 ) for each of the 4 different time intervals $(-4$, $-1,1$, and $4 \mathrm{wk}$ ). Two different comparisons were performed, a cross-sectional and a longitudinal evaluation. The cross-sectional evaluation compared healthy with diseased cows at the same time points whereas the longitudinal evaluation tracked the metabolic changes in the healthy cows over the 4 time points and the diseased cows over the same time points. We will discuss the results of the cross-sectional study first and the longitudinal study second.

\section{Univariate Statistical Analysis}

A univariate analysis using a $t$-test was conducted for 120 metabolites for the healthy controls and diseased cows. The results of significantly $(P<0.05)$ regulated metabolites in healthy versus diseased cows at 4 time points during the transition period are shown in Table 4. The concentrations of 5 metabolites, carnitine $(\mathrm{C} 0)$, propionyl carnitine (C3), lysophosphatidylcholine acyl C14:0 (lysoPCaC14:0), lysoPhosphatidylcholine acyl C18:2 (lysoPCaC18:2), and valerylcarnitine (C5), were elevated in diseased cows as compared with the healthy controls 4 wk before parturition. Similarly, the concen- 
Table 3. List of metabolites identified and quantified by direct flow injection MS/MS

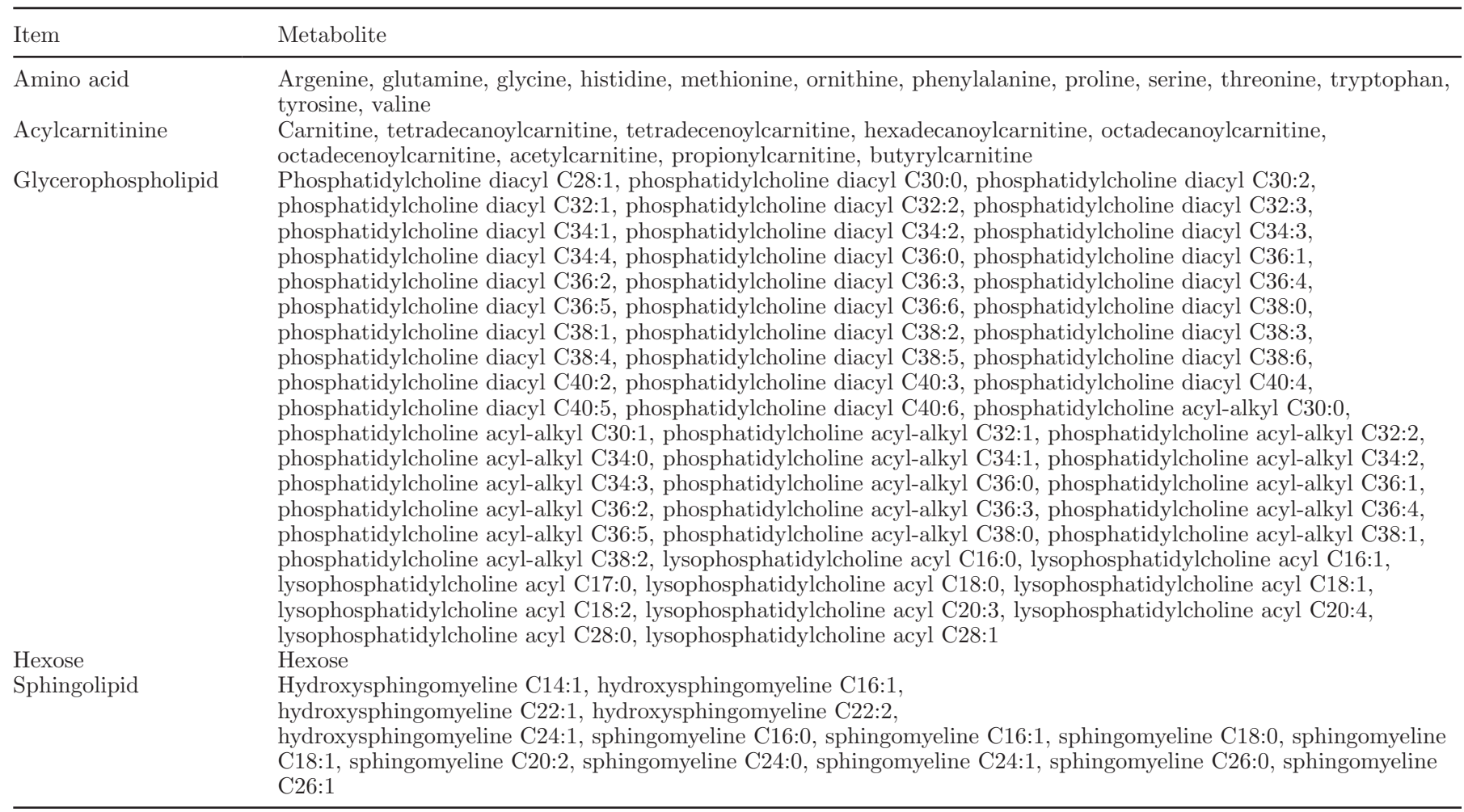

trations of phosphatidylcholine acyl-alkyl C42:4 (PC ae C42:4), phosphatidylcholine diacyl C42:4 (PC aac42:6), carnitine (C0), lysophosphatidylcholine acyl C14:0 (lyso PC 14:0), and lysophosphatidylcholine acyl C14:0 acyl C28:0 (lyso PC a 28:0) were increased in diseased cows as compared with their healthy counterparts $1 \mathrm{wk}$ before and $1 \mathrm{wk}$ after parturition. However, at $4 \mathrm{wk}$ after parturition, we observed lowered concentrations of metabolites carnitine $(\mathrm{C} 0)$, phosphatidylcholine diacyl C42:6 (PC aa C42:6), phosphatidylcholine acyl-alkyl C42:4 (PC ae C42:4), and lysophosphatidylcholine acyl $\mathrm{C} 28: 0$ (lyso PCa C28:0) in diseased cows as compared with the healthy ones.

\section{Multivariate Analysis on Plasma Metabolites at -4 and -1 Wk Before Parturition}

When healthy cows were compared with not-yet-diseased cows (those that eventually developed mastitis, metritis, laminitis, and retained placenta) at $-4 \mathrm{wk}$ using PCA, 2 clear clusters could be seen (Figure 1a). Furthermore, 3 metabolites accounted for most of the observed separation, carnitine ( $\mathrm{C} 0)$, propionyl carnitine (C3), and lysophosphatidylcholine acyl C14:0 (lysoP$\mathrm{CaC} 14$; a lysophospholipid). These compounds were elevated in the diseased animals and had VIP scores of 4,4 , and 6 , respectively, indicating a significant con- tribution in separating healthy and diseased animals. The remaining blood plasma metabolites showed much lower VIP scores $(<2)$ with minimal contribution to the variation that separated the healthy and diseased groups (Figure 1, b and c). A ROC plot showing the performance of the top 5 metabolites in predicting which cows will develop periparturient diseases at -4 wk using a standard support vector machine model is shown in Figure 1d. The AUC for this curve is 0.950 , which indicates that these 5 biomarkers have very good predictive ability. Interestingly, at $-4 \mathrm{wk}$, none of the 12 cows exhibited any outward signs of disease. This suggests that, metabolically, the disease processes must begin quite early. More interestingly, these results suggest that the biomarker model developed here could be used to predict which cows will develop postpartum diseases 4 wk before they actually develop the conditions.

When healthy cows were compared with not-yetdiseased cows at $-1 \mathrm{wk}$, the PCA plot once again showed a clear separation (Figure 2a). In this case, 2 blood plasma metabolites [phosphatidylcholine acylalkyl C42:4 (PCec42:4) and phosphatidylcholine diacyl C42:6 (PCaaC42:6)] with VIP scores of 4.5 and 3.4 contributed most significantly to the separation between the 2 groups. Whereas the discrimination between the 2 groups is almost as strong at $-4 \mathrm{wk}$, it is notable 
Table 4. Concentrations of plasma metabolites in healthy control and diseased cows at 4 time points $(-4,-1,1$, and 4 wk) during the transition period as determined by direct-flow injection-MS/MS (mean $\pm \mathrm{SD} ; \mathrm{n}=6$ )

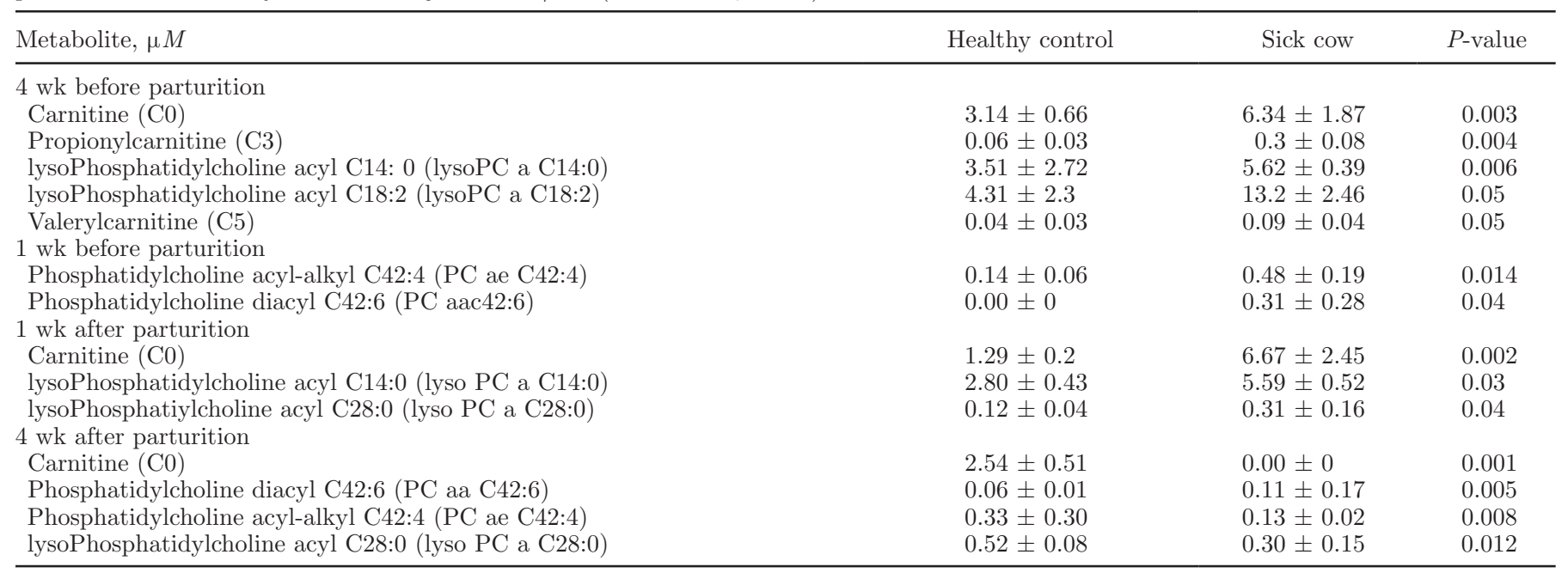

that the metabolites most responsible for the separation are different (Figure 2, b and c). Only 1 metabolite (lysoPCaC14) identified from the -4 wk cohort retained a significant VIP score (1.8) at 1 wk before calving. A ROC plot showing the performance of the top 2 metabolites in predicting which cows will develop periparturient diseases at -1 wk using a support vector machine model is shown in Figure 2d. The AUC for this curve is 0.845 , which indicates that these 2 biomarkers have very good predictive ability. As before, at 1 wk before calving none of the 12 cows exhibited any outward signs of disease.

\section{Multivariate Analyses at 1 and 4 Wk After Parturition}

When healthy cows were compared with diseased cows just 1 wk after calving, our multivariate statistical analysis (PCA and PLS-DA) revealed a strikingly consistent separation between the healthy and diseased cows (Figure 3, a and b). Moreover, 3 metabolites (C0, lysoPCaC14, and lysoPCa28) with corresponding VIP scores of 2.7, 4.9, and 6.8 contributed most significantly to the observed separation (Figure 3c). Interestingly, carnitine $(\mathrm{C} 0)$ and lysoPCaC14 are the same plasma metabolites responsible for separating the 2 groups at 4 wk before parturition. As with the other high-importance metabolites identified in the earlier time points, these metabolites showed elevated concentrations in diseased cows as compared with the healthy ones.

When healthy cows were compared with diseased cows at 4 wk after parturition, PCA and PLS-DA once again revealed a clear separation between healthy and diseased animals (Figure 4, a and b). At this point, all 6 diseased cows were exhibiting or had shown clear symptoms of one or more of the 4 periparturient diseases. Four metabolites (C0, PC aa $\mathrm{C} 42: 6, \mathrm{PC}$ ae $\mathrm{C} 42: 4$, and lysoPCaC28) with strong VIP scores of 4.5 , $3.8,2.7$, and 1.8, respectively, discriminated strongly between the healthy and diseased cows. It is interesting to note that throughout the 8-wk study, 2 blood plasma metabolites ( $\mathrm{C} 0$ and lysoPCaC14) appeared to play a consistent role in distinguishing between healthy and diseased cows (Figure 4c).

\section{Time Course Disease Trajectory During the Transition Period}

The principal component score plot for the interaction of healthy and diseased groups with respect to the significantly altered metabolites at 4 time points during the transition period is shown in Figure 5. It is evident from this plot that the trajectories are very different with the 2 groups of animals (in metabolic PCA space). In particular, the direction of change in PCA space for $\mathrm{PC} 1$ (56.1\%) is perhaps distinct, with the diseased cows having higher concentrations of metabolites at -4 and 1 wk than their healthy counterparts; whereas this trend abruptly switched at 4 wk, when the concentrations of metabolites for diseased cows declined as opposed to a rising trend in healthy cows. For the PC2 (24.3\%), the diseased and healthy groups showed different metabolites concentration patterns over the 4 time points around calving $(-4,-1,1$, and 4 wk). It is also worth noting that the trend of metabolite concentrations in diseased cows declined after calving as opposed to increasing trends for the healthy cows. This indicates the deviation of metabolism of sick cows from the healthy status both before and after the clinical manifestation of the disease. 
a

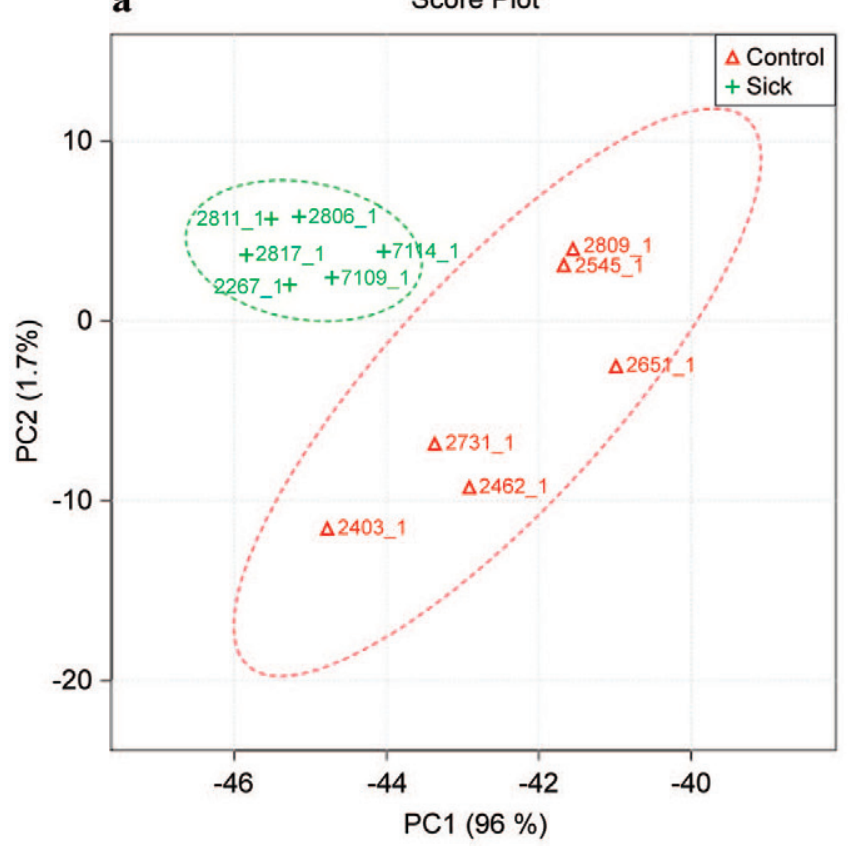

b

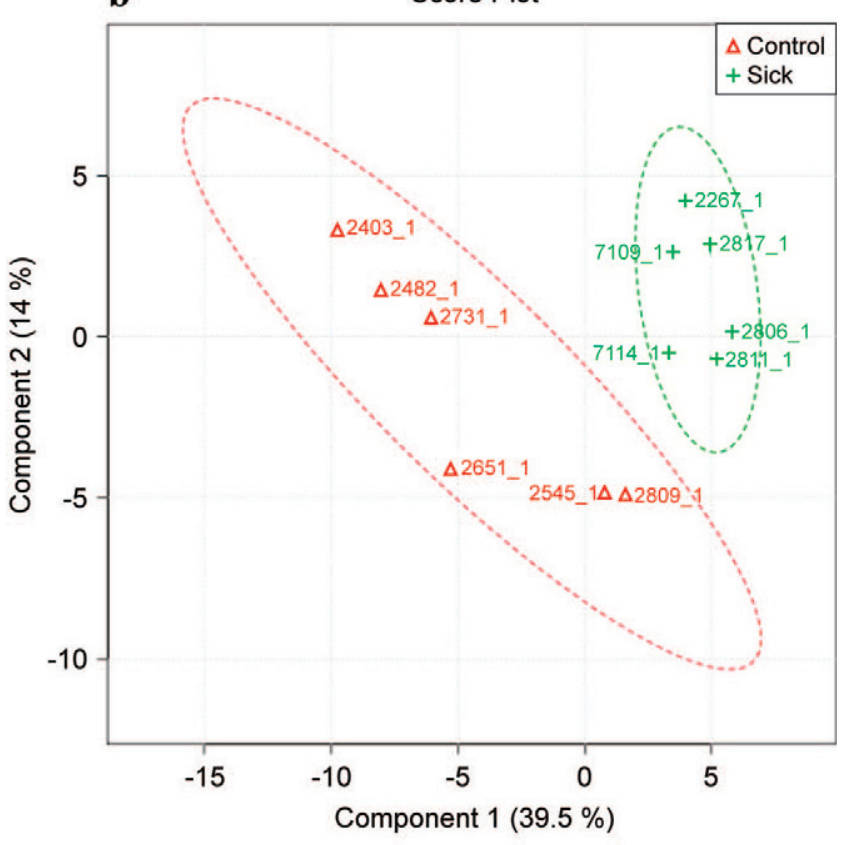

d

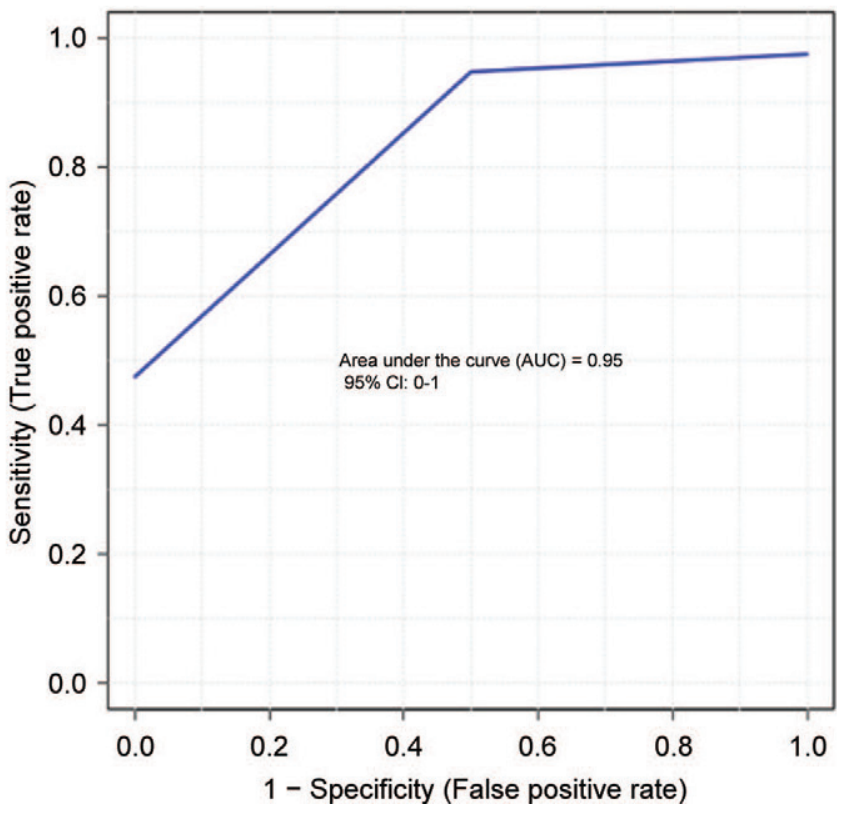

Figure 1. (a) Principal component (PC) analysis of 6 control and 6 diseased cows at 4 wk before parturition. (b) Partial least squares-discriminant analysis showing 2 clusters for 2 groups and (c) metabolites ranked by variable importance in projection (VIP). (d) Receiver-operator characteristic curve of 6 control and 6 diseased cows at -4 wk before parturition for the top 3 plasma metabolites. Color version available in the online PDF.

\section{Plasma Metabolites as Predictive Biomarkers of Disease State}

As noted earlier, all the periparturient pathologies (mastitis, metritis, laminitis, and retained placenta) observed were grouped into one category (diseased).
Whereas these pathologies affect different organs or tissues, they are interrelated, which suggested that some early stage metabolic commonalities for those conditions may exist. Using quantitative MS-based metabolomics of bovine blood plasma we found that healthy animals could indeed be clearly and consis- 

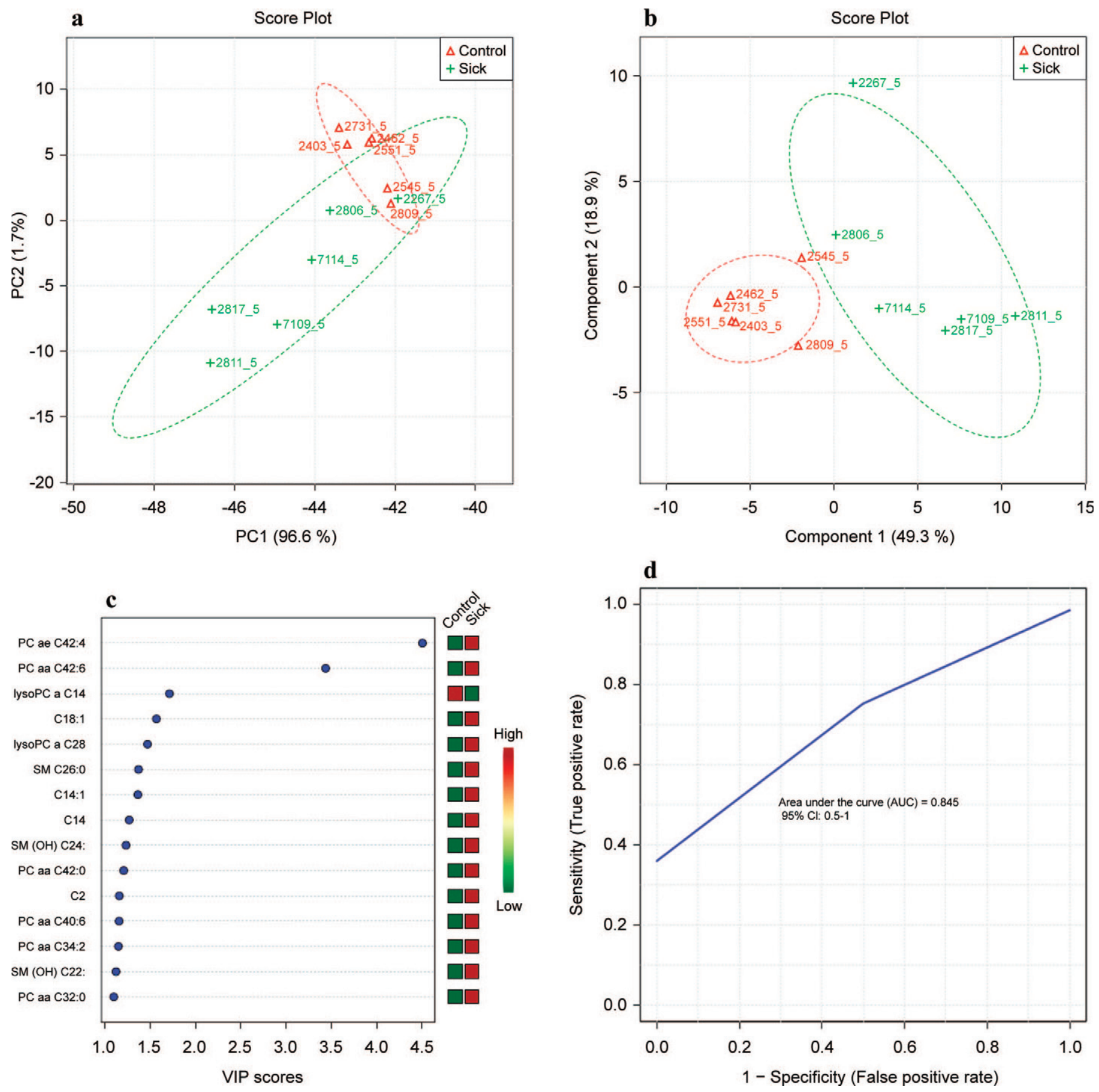

Figure 2. (a) Principal component (PC) analysis of 6 control and 6 diseased cows at -1 wk before parturition. (b) Partial least squares-discriminant analysis showing 2 clusters for 2 groups (c) and metabolites ranked by variable importance in projection (VIP). (d) Receiver-operator characteristic curve of 6 control and 6 diseased cows at 1 wk before parturition for the top 2 plasma metabolites. Color version available in the online PDF.

tently distinguished from the diseased ones or soonto-be diseased animals. More interestingly, we found that these differences were evident up to 4 wk before the animals exhibiting any outward signs of the disease(s). These results suggest that some surprising metabolic commonalities exist between these very diverse postpartum conditions. Using a longitudinal or time-course PCA approach, we also observed that healthy animals exhibited a much different or more restrained metabolic change through the transition period compared with animals that would later go on to develop postpartum diseases. 

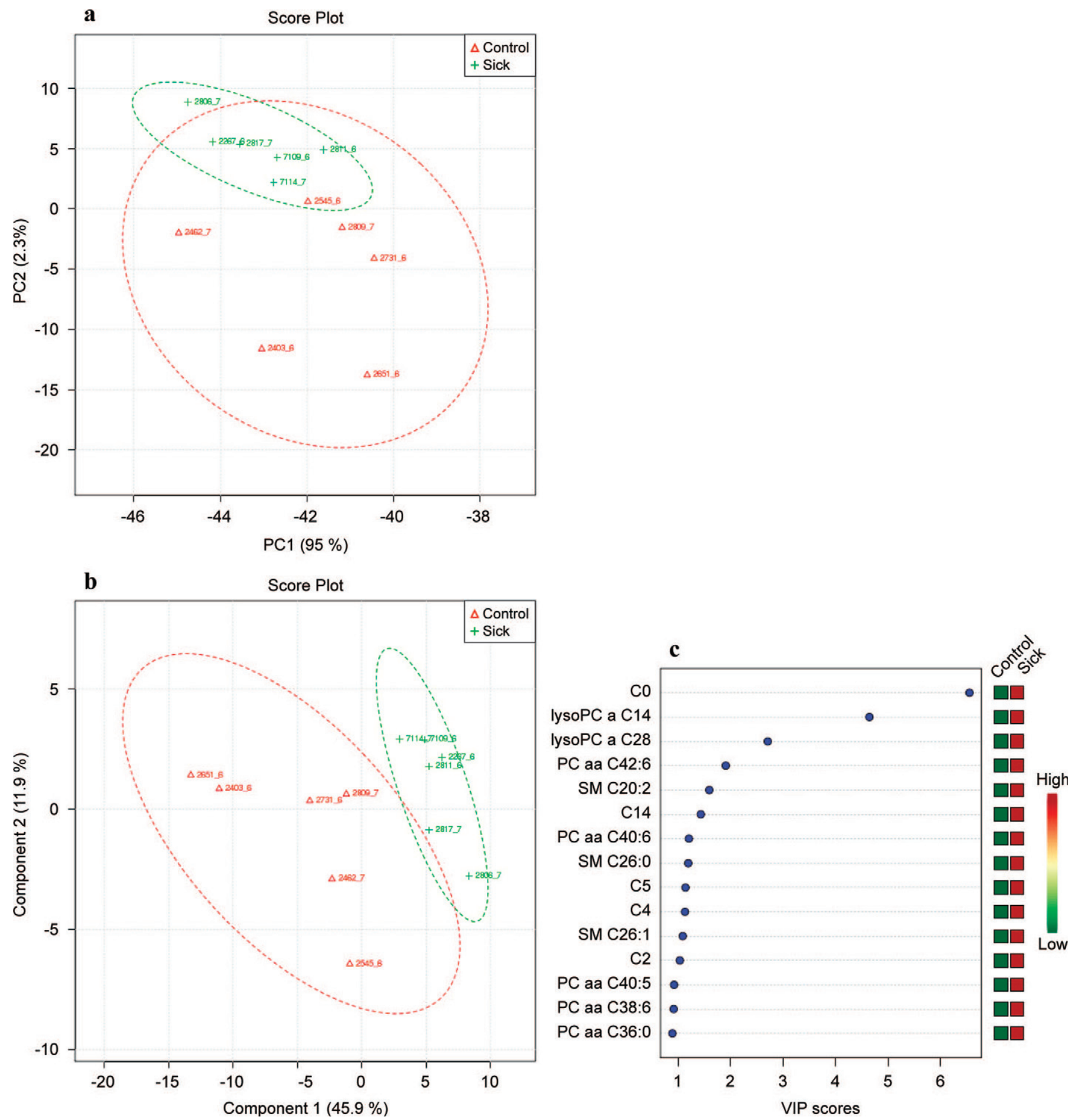

Figure 3. (a) Principal component (PC) analysis of 6 control and 6 diseased cows at 1 wk after parturition. (b) Partial least squares-discriminant analysis showing 2 clusters for 2 groups and (c) metabolites ranked by variable importance in projection (VIP). Color version available in the online PDF.

Multivariate analysis and modeling (PCA, PLS-DA, VIP, and ROC curve analysis) showed that 5 plasma metabolites, such as carnitine (C0), propionyl carni- tine (C3), lysophosphatidylcholine acyl C14:0 (lysoPCaC14), PCec42:4, and PCaaC42:6, could be used to generate at least 2 highly reliable $(\mathrm{AUC}=0.95,0.845)$ 

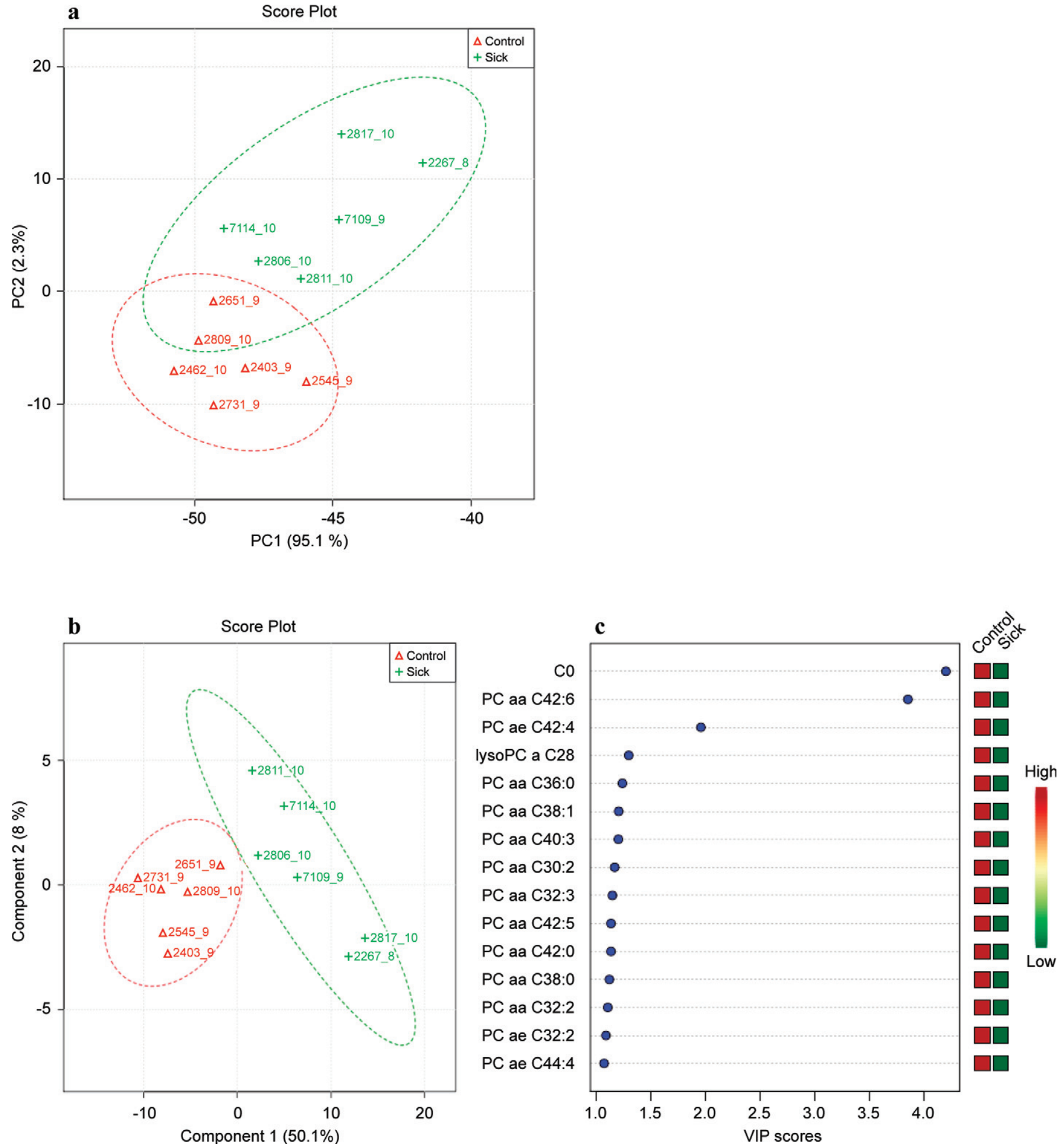

Figure 4. (a) Principal component (PC) analysis of 6 control and 6 diseased cows at 4 wk after parturition. (b) Partial least squares-discriminant analysis showing 2 clusters for 2 groups and (c) metabolites ranked by variable importance in projection (VIP). Color version available in the online PDF. 
a

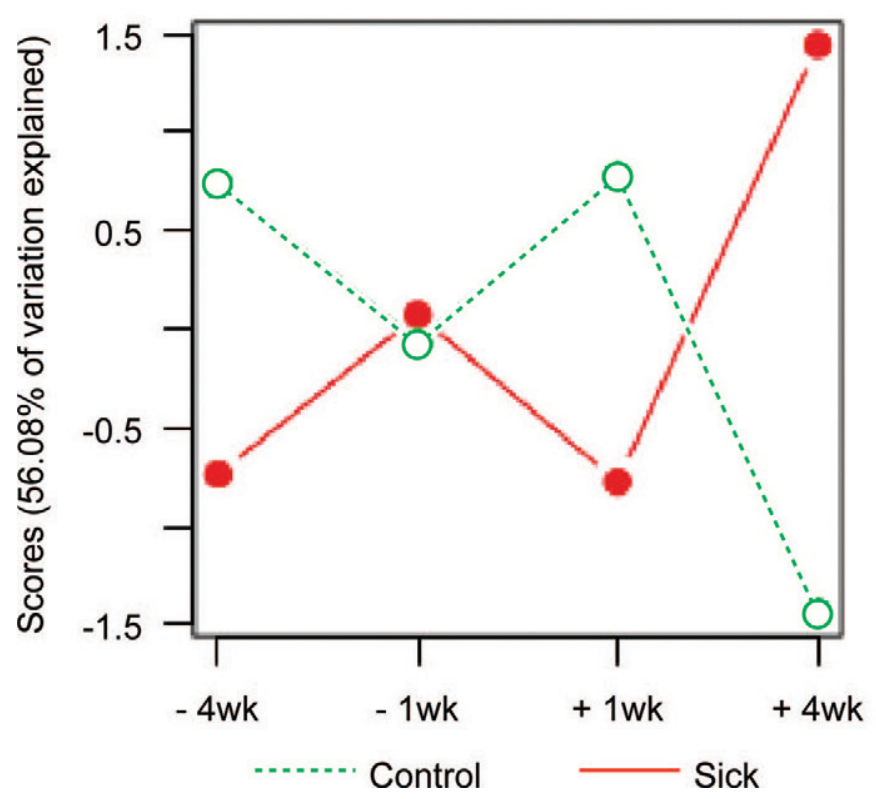

b

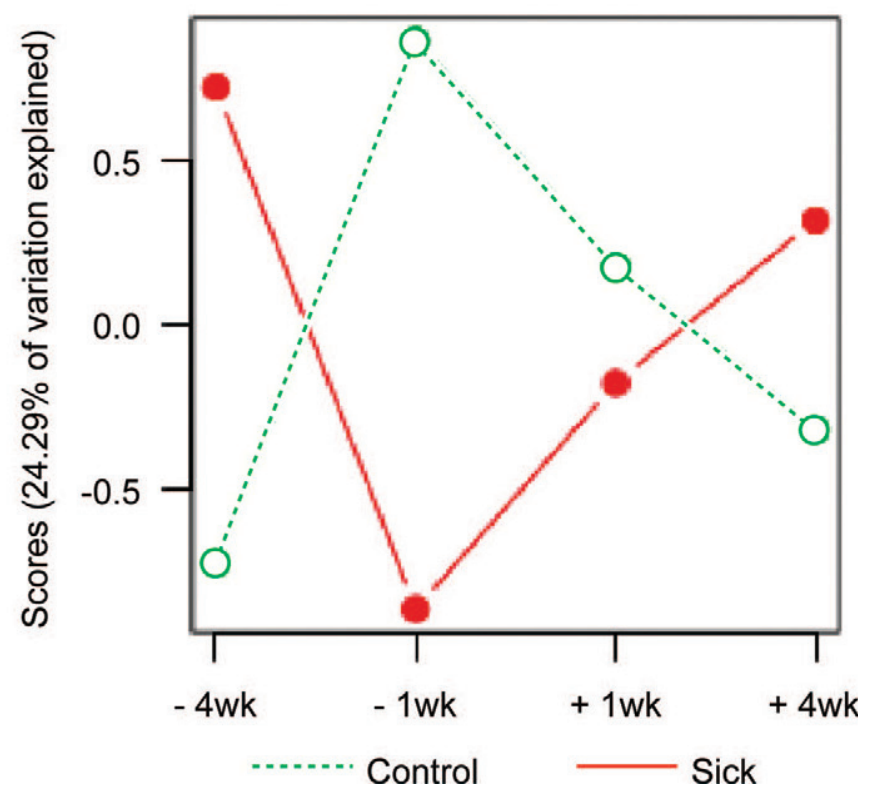

Figure 5. Time-course principal component analysis (PCA) plots comparing the disease trajectory for component 1 (a) and component 2 (b). The PCA plot has been constructed for significantly different metabolites between healthy and diseased cows over the 4 different time points. Color version available in the online PDF.

biomarker models for predicting which cows would develop one or more periparturient disorders. These biomarkers could be used at -4 and -1 wk before calving and up to $4 \mathrm{wk}$ before the clinical onset of the pathologies. That these changes were so visible, even at -4 wk before calving, suggests that the detection or prediction envelope could be pushed back even further-perhaps to 6 to 8 wk or more.

The fact that these 4 very different disease states clustered together metabolically is somewhat surprising. Certainly if more samples could have been acquired it might have been possible to use metabolic profiling to distinguish between these conditions or to develop a disease-specific metabolic signature. Our data suggest that mastitis, metritis, laminitis, and retained placenta seem to share some common metabolic underpinnings. This is a very interesting result and could help shed some light on understanding how and why these conditions develop. In this regard it is perhaps useful to discuss what role some of these 5 key metabolites may have in metabolism, signaling, or immunological responses.

\section{Plasma Metabolites Dysregulation and Pathophysiology of Periparturient Disorders}

Carnitine and propionylcarnitine are acylcarnitines. Carnitine and related acylcarnitines help shuttle FA from the cytoplasm across the inner mitochondrial membrane into the mitochondrial matrix (Stanley et al., 1992). Longer chain acylcarnitines play an important role in the lipid $\beta$-oxidation process. Conversely, short-chain acylcarnitines tend to be involved in AA metabolism, particularly in branched-chain AA degradation (Roe et al., 2000). Carnitines and acylcarnitines are often the by-products of cells that have nuclei and mitochondria (such as muscles and white blood cells). Mammalian red blood cells or erythrocytes, which constitute $99 \%$ of the cells in blood, have no nucleus and no mitochondria. Therefore elevated levels of carnitines and other short-chain acylcarnitines in plasma can only arise from the activity or lysis of white blood cells or through the lysis or death of other nucleated, metabolically active cells (found in muscle, liver, kidney, or other large organs, including the uterus). In other words, acylcarntitines are markers of either immune activation or organ dysfunction. Several recent metabolomic studies have identified carnitine and acylcarnitines as useful biomarkers for many inflammatory or autoimmune human diseases (Guo et al., 2012; Sampey et al., 2012; van der Kloet et al., 2012; Frye et al., 2013). These data suggest that carnitine or acylcarnitines are more likely general, rather than specific markers, of general inflammatory processes. Given that mastitis and metritis typically arise from bacterial infections or bacterially induced inflammation, and laminitis and retained pla- 
centa are associated with inflammation and the latter potentially with infection, the appearances of increased serum carnitine and acylcarnitine levels seems entirely reasonable.

Lysophosphatidcholines are produced from cell membrane-derived phosphatidylcholine following the hydrolysis of phosphatidylcholine by phospholipase $\mathrm{A}_{2}$. Different lysophospholipids are distinguished by the lengths and saturation of their acyl chains (McKean et al., 1981). Lysophospholipids are often used as signaling molecules by eukaryotic cells. In particular, lysophosphatidcholine's regulatory role in immune functions and its association with inflammatory processes has been well documented by many authors (Iuliano et al., 1997; Koh et al., 2000; Romero et al., 2000; Kabarowski et al., 2002). Specifically, lysophosphatidcholine promotes increased monocyte chemotaxis (Jing et al., 2000) and enhances macrophage activation (Yamamoto et al., 1991). The increased plasma concentrations of lysophosphatidcholines we observed in diseased cows up to 4 wk before the onset of any clinical signs of the pathologic condition might correlate with an increased demand for an immunoregulatory ligand at the earliest stages of the disease. Given that many of these postpartum diseases are associated with bacterial infections or bacterially induced inflammation (mastitis, metritis, and retained placenta), an elevated immune response would certainly be expected.

Phosphatidylcholine acyl-alkylC42:4 and phosphatidylcholine diacyl C42:6 are 2 phospholipids that were found to be most responsible for separating diseased cows from healthy cows 1 wk before parturition. It is not clear whether these phospholipids are of bacterial or bovine origin, although the high levels of acyl desaturation suggest they are more likely of bovine origin. It is known that phospholipid turnover in mammals can be reduced by certain kinds of pathogenic bacteria through the inhibition of phospholipase $\mathrm{A}_{2}$ (Duan et al., 2001). Furthermore, these same bacteria can also increase production of phospholipids. Interestingly, an increase in phospholipids was observed among dairy cows 1 wk before parturition. Therefore, one might expect that the infection of dairy cows (i.e., during metritis, mastitis, and retained placenta) with multiple bacterial species during parturition could lead to some plasma lipid dysregulation.

Despite the specificity, reproducibility, and noninvasiveness of metabolomics to identify potential predictive biomarkers for the periparturient pathologies in dairy cows, the cost and ease of sample analysis may limit its universal use by dairy farmers. Therefore, after validating these potential biomarkers in large cohorts of animals, it appears important to develop a more con- venient or accessible dip-stick assay so that these tests could be easily used by dairy farmers.

\section{CONCLUSIONS}

In summary, we have identified 3 compounds, carnitine ( $\mathrm{C} 0)$, propionyl carnitine (C3), and lysophosphatidylcholine acyl C14:0 (lysoPC a C14:0), that may predict disease state up to 4 wk before the apparent or clinical onset of the disease. Conversely, 2 plasma metabolites phosphatidylcholine acyl-alkylC42:4 (PC ae $\mathrm{C} 42: 4)$ and phosphatidylcholine diacyl C42:6 (PC aa $\mathrm{C} 42: 6)$, may indicate disease a week before disease onset. In the current study, we showed the potential for a multimetabolite panel to predict periparturient diseases. However, similar to any set of candidate biomarkers, these biomarker profiles will need to be further validated using a much larger cohort of animals. This validation process is now being completed and preliminary data support that these markers are robust. If these markers are successfully validated it will also be necessary to develop a more convenient or accessible dip-stick assay so that these tests could be easily used by dairy farmers.

\section{ACKNOWLEDGMENTS}

Financial support for this work was provided by Genome Alberta (Calgary, Alberta, Canada), Alberta Livestock and Meat Agency Ltd. (Edmonton, Alberta, Canada), and the Natural Sciences and Engineering Research Council of Canada (Ottawa, Ontario, Canada). We are also grateful to the technical staff of Dairy Research and Technology Centre at the University of Alberta for their help in caring for and monitoring the cows used in this study.

\section{REFERENCES}

Ametaj, B. N., Q. Zebeli, S. Iqbal, and S. M. Dunn. 2012. Meeting the challenges of improving health in periparturient dairy cows. Adv. Dairy Technol. 24:287-317.

Ametaj, B. N., Q. Zebeli, F. Saleem, N. Psychogios, M. J. Lewis, S. M. Dunn, J. Xia, and D. S. Wishart. 2010. Metabolomics reveals unhealthy alterations in rumen metabolism with increased proportion of cereal grain in the diet of dairy cows. Metabolomics 6:583-594.

Bahado-Singh, R. O., R. Akolekar, R. Mandal, E. Dong, J. Xia, M. Kruger, D. S. Wishart, and K. Nicolaides. 2012. Metabolomics and first-trimester prediction of early-onset preeclampsia. J. Matern. Fetal Neonatal Med. 25:1840-1847.

Bahado-Singh, R. O., R. Akolekar, R. Mandal, E. Dong, J. Xia, M. Kruger, D. S. Wishart, and K. Nicolaides. 2013. Metabolomic analysis for first-trimester Down syndrome prediction. Am. J. Obstet. Gynecol. 208:371.

Bigras-Poulin, M., A. H. Meek, and S. W. Martin. 1990. Interrelationships among health problems and milk production from consecu- 
tive lactations in selected Ontario Holstein cows. Prev. Vet. Med. $8: 15-24$.

Buch, L. H., A. C. Sørensen, J. Lassen, P. Berg, J. Å. Eriksson, J. H. Jakobsen, and M. K. Sørensen. 2011. Hygiene-related and feedrelated hoof diseases show different patterns of genetic correlations to clinical mastitis and female fertility. J. Dairy Sci. 94:1540-1551.

Canadian Council on Animal Care. 1993. Guide to the Care and Use of Experimental Animals. Vol. 1. E. D. Olfert, B. M. Cross, and A. A. McWilliam, ed. Canadian Council on Animal Care, Ottawa, ON, Canada.

Correa, M. T., H. N. Erb, and J. Scarlett. 1993. Path analysis for seven postpartum disorders of Holstein cows. J. Dairy Sci. 76:13051312 .

Curtis, C. R., H. N. Erb, C. J. Sniffen, R. D. Smith, P. A. Powers, M. C. Smith, M. E. White, R. B. Hillman, and E. J. Pearson. 1983. Association of parturient hypocalcemia with eight periparturient disorders in Holstein cows. J. Am. Vet. Med. Assoc. 183:559-561.

Duan, L., H. Gan, J. Arm, and H. G. Remold. 2001. Cytosolic phospholipase A2 participates with TNF-alpha in the induction of apoptosis of human macrophages infected with Mycobacterium tuberculosis H37Ra. J. Immunol. 166:7469-7476.

Enjalbert, F., M. C. Nicot, C. Bayourthe, and R. Moncoulon. 2001. Ketone bodies in milk and blood of dairy cows: Relationship between concentrations and utilization for detection of subclinical ketosis. J. Dairy Sci. 84:583-589.

Frye, R. E., S. Melnyk, and D. F. Macfabe. 2013. Unique acyl-carnitine profiles are potential biomarkers for acquired mitochondrial disease in autism spectrum disorder. Transl. Psychiatry 3:e220.

Guo, Y., X. Wang, L. Qiu, X. Qin, H. Liu, Y. Wang, F. Li, G. Chen, G. Song, S. Guo, and Z. Li. 2012. Probing gender-specific lipid metabolites and diagnostic biomarkers for lung cancer using Fourier transform ion cyclotron resonance mass spectrometry. Clin. Chim. Acta 414:135-141.

Iuliano, L., D. Pratico, D. Ferro, V. Pittoni, G. Valesini, J. Lawson, G. A. FitzGerald, and F. Violi. 1997. Enhanced lipid peroxidation in patients positive for antiphospholipid antibodies. Blood 90:3931-3935.

Jing, Q., S. M. Xin, W. B. Zhang, P. Wang, Y. W. Qin, and G. Pei. 2000. Lysophosphatidylcholine activates p38 and p42/44 mitogenactivated protein kinases in monocytic THP-1 cells, but only p38 activation is involved in its stimulated chemotaxis. Circ. Res. $87: 52-59$

Kabarowski, J. H., Y. Xu, and O. N. Witte. 2002. Lysophosphatidylcholine as a ligand for immunoregulation. Biochem. Pharmacol. 64:161-167.

Klein, M. S., N. Buttchereit, S. P. Miemczyk, A. K. Immervoll, C. Louis, S. Wiedemann, W. Junge, G. Thaller, P. J. Oefner, and W. Gronwald. 2012. NMR metabolomic analysis of dairy cows reveals milk glycerophosphocholine to phosphocholine ratio as prognostic biomarker for risk of ketosis. J. Proteome Res. 11:1373-1381.

Kloosterman, P. 2007. Laminitis-Prevention, diagnosis and treatment. Adv. Dairy Technol. 19:157-166.

Koh, J. S., Z. Wang, and J. S. Levine. 2000. Cytokine dysregulation induced by apoptotic cells is a shared characteristic of murine lupus. J. Immunol. 165:4190-4201.

LeBlanc, S. 2010. Monitoring metabolic health of dairy cattle in the transition period. J. Reprod. Dev. 56(Suppl.):S29-S35.

McKean, M. L., J. B. Smith, and M. J. Silver. 1981. Formation of lysophosphatidylcholine by human platelets in response to thrombin. Support for the phospholipase A2 pathway for the liberation of arachidonic acid. J. Biol. Chem. 256:1522-1524.

Ospina, P. A., D. V. Nydam, T. Stokol, and T. R. Overton. 2010. Evaluation of nonesterified fatty acids and beta-hydroxybutyrate in transition dairy cattle in the northeastern United States: Critical thresholds for prediction of clinical diseases. J. Dairy Sci. 93:546-554.
Piechotta, M., A. Sander, J. Kastelic, R. Wilde, M. Heppelmann, B. Rudolphi, H. Schuberth, H. Bollwein, and M. Kaske. 2012. Short communication: Prepartum plasma insulin-like growth factor-I concentrations based on day of insemination are lower in cows developing postpartum diseases. J. Dairy Sci. 95:1367-1370.

Roe, D. S., C. R. Roe, M. Brivet, and L. Sweetman. 2000. Evidence for a short-chain carnitine-acylcarnitine translocase in mitochondria specifically related to the metabolism of branched-chain amino acids. Mol. Genet. Metab. 69:69-75.

Romero, F. I., M. A. Khamashta, and G. R. Hughes. 2000. Lipoprotein(a) oxidation and autoantibodies: A new path in atherothrombosis. Lupus 9:206-209.

Saleem, F., B. N. Ametaj, S. Bouatra, R. Mandal, Q. Zebeli, S. M. Dunn, and D. S. Wishart. 2012. A metabolomics approach to uncover the effects of grain diets on rumen health in dairy cows. J. Dairy Sci. 95:6606-6623.

Sampey, B. P., A. J. Freemerman, J. Zhang, P. F. Kuan, J. A. Galanko, T. M. O'Connell, O. R. Ilkayeva, M. J. Muehlbauer, R. D. Stevens, C. B. Newgard, H. A. Brauer, M. A. Troester, and L. Makowski. 2012. Metabolomic profiling reveals mitochondrial-derived lipid biomarkers that drive obesity-associated inflammation. PLoS ONE $7: \mathrm{e} 38812$.

Sheldon, I. M., G. S. Lewis, S. LeBlanc, and R. O. Gilbert. 2006. Defining postpartum uterine disease in cattle. Theriogenology 65:1516-1530.

Stanley, C. A., D. E. Hale, G. T. Berry, S. Deleeuw, J. Boxer, and J. P. Bonnefont. 1992. Brief report: A deficiency of carnitine-acylcarnitine translocase in the inner mitochondrial membrane. N. Engl. J. Med. 327:19-23.

Sundekilde, U. K., P. D. Frederiksen, M. R. Clausen, L. B. Larsen, and H. C. Bertram. 2011. Relationship between the metabolite profile and technological properties of bovine milk from two dairy breeds elucidated by NMR-based metabolomics. J. Agric. Food Chem. 59:7360-7367.

Sundekilde, U. K., N. Poulsen, L. B. Larsen, and H. C. Bertram. 2013. Nuclear magnetic resonance metabonomics reveals strong association between milk metabolites and somatic cell count in bovine milk. J. Dairy Sci. 96:290-299.

van der Kloet, F. M., F. W. Tempels, N. Ismail, R. van der Heijden, P. T. Kasper, M. Rojas-Cherto, R. van Doorn, G. Spijksma, M. Koek, J. van der Greef, V. P. Makinen, C. Forsblom, H. Holthofer, P. H. Groop, T. H. Reijmers, and T. Hankemeier. 2012. Discovery of early stage biomarkers for diabetic kidney disease using msbased metabolomics. Metabolomics 8:109-119.

Wishart, D. S. 2010. Computational approaches to metabolomics. Methods Mol. Biol. 593:283-313.

Xia, J., D. I. Broadhurst, M. Wilson, and D. S. Wishart. 2013. Translational biomarker discovery in clinical metabolomics: An introductory tutorial. Metabolomics 9:280-299.

Xia, J., N. Psychogios, N. Young, and D. S. Wishart. 2009. MetaboAnalyst: A web server for metabolomic data analysis and interpretation. Nucleic Acids Res. 37:W652-660.

Yamamoto, N., S. Homma, and I. Millman. 1991. Identification of the serum factor required for in vitro activation of macrophages. Role of vitamin $\mathrm{D}_{3}$-binding protein (group specific component, $\mathrm{Gc}$ ) in lysophospholipid activation of mouse peritoneal macrophages. J. Immunol. 147:273-280.

Zadoks, R. N., J. R. Middleton, S. McDougall, J. Katholm, and Y. H. Schukken. 2011. Molecular epidemiology of mastitis pathogens of dairy cattle and comparative relevance to humans. J. Mammary Gland Biol. Neoplasia 16:357-372.

Zebeli, Q., S. M. Dunn, and B. N. Ametaj. 2011. Perturbations of plasma metabolites correlated with the rise of rumen endotoxin in dairy cows fed diets rich in easily degradable carbohydrates. J. Dairy Sci. 94:2374-2382. 